\title{
Energy Dispersive Spectrometry at Wavelength Precision
}

\author{
Nicholas W. M. Ritchie*, Jeff Davis* \& Dale E. Newbury* \\ * National Institute of Standards and Technology, 100 Bureau Drive, MS: 8371, Gaithersburg, MD \\ 20899-8371
}

Wavelength spectrometry (WDS) is generally considered to be the gold standard for electron-excited $\mathrm{x}$-ray microanalytical measurements in an electron beam instrument. The high resolving power of $\mathrm{x}$ ray diffraction means that peak-to-background ratios can be high and that almost all combinations of $\mathrm{x}$-ray transition lines can be resolved without resorting to peak deconvolution. Furthermore because of the narrow energy bandpass of the wavelength spectrometer, the output count rate for a specific energy is not limited by the presence of x-rays at other energies. However, energy dispersive spectrometers are not without advantages. The x-ray collection efficiency can be orders-ofmagnitude higher so lower electron doses can be used. Furthermore, the full spectrum is always collected in a single measurement eliminating the need for multiple spectrometers, moving or flipping spectrometer crystals and many of the other complexities associated with wavelength spectrometers. It has long been suggested that that energy dispersive spectrometers can selectively replace wavelength spectrometers[1]. The commercial availability of silicon drift detectors (SDD) and high throughput pulse processing which have become available in the past decade strengthens this case[2].

We compared the precision of WDS measurements with measurements made with a state-of-theart SDD on a modern microprobe to determine which detector is optimal for many different classes of measurements. For the most rigorous test, we compared the WDS k-ratio to the SDD k-ratio (the ratio of the specimen to standard intensities) for spectra collected for similar durations. Comparing k-ratios encapsulates fully the dependence of the precision on the detector. However, we learned that there are circumstances when the k-ratios are not comparable because the SDD measurement windows extends across absorption edges whereas the much narrower WDS measurement does not. One such case is the $\mathrm{Cu} \mathrm{L}$ in which the complex edge structure makes comparison of the intensity in the $\mathrm{Cu}$ L-family (as measured by SDD) incomparable with the $\mathrm{Cu} \mathrm{L}_{3}-\mathrm{M}_{5}$ (as measured by WDS)[3]. Fortunately, this concern is unusual and k-ratios are usually a good quantity to compare.

We considered relatively simple measurements in which the material constituents were present in major proportions (mass fraction $>0.10$ ) and the peaks were well resolved even on the SDD. We also examined a series of more challenging problems. We considered severe overlaps in which one element was totally occluded by the other like the $\mathrm{Ba} \mathrm{L} / \mathrm{Ti} \mathrm{K}$ overlap and the $\mathrm{Pb} / \mathrm{S} / \mathrm{Mo}$ overlaps, including situations with high concentration ratios. We investigated materials in which one element was present at below 0.01 mass fraction. We also investigated low energy x-ray in which the more complicated structure of the bremsstralung background makes peak convolution of the SDD spectra more challenging. The results of these studies have been surprising in many regards not the least of which is that SDD-EDS can perform as well as WDS for many problems, but at significantly lower dose.

One particularly compelling data set considered a series of $\mathrm{Ba} / \mathrm{Ti}$ glasses and standards. These glasses varied from 0.017 mass fraction to 0.201 mass fraction titanium and 0.3 to 0.6 mass fraction barium. This represents a classic challenge for EDS analysis but one which the one-minute, $5,000,000$ count SDD spectra were easily able to handle. Figure 1 shows the results. The error bars 
represent one standard-deviation of the eight measurements taken at different points on the sample. The SDD and WDS data was collected simultaneously to mitigate any sample inhomogeneity biases.

Our measurements demonstrate that for many practical cases that silicon drift detectors are capable of similar or better precision than wavelength spectrometers including some very challenging cases such as the $\mathrm{Ba} \mathrm{L} \alpha$ and $\mathrm{Ti} \mathrm{K} \alpha$ separated by $41 \mathrm{eV}$ and with elemental ratios as high as $24: 1$.

[1] S. J. B. Reed \& N. G. Ware, Quantitative electron microprobe analysis using a lithium drifted silicon detector, X-ray Spectrom. 2 (1973) p 69-73

[2] E. Gatti \& P. Rehak, Semiconductor drift chamber - An application of a novel charge transport scheme, Nucl. Inst. and Meth. Sec. V, 225 Issue 3 (1984) pp 608-614

[3] J. Kawai, K. Nakajumi, Y. Gohshi, Copper L $\beta / L \alpha X$-ray emission intensity ratio of copper componds and alloys, Spectrochimica Acta, 48B number 1 (1993), pp 1281-1290.

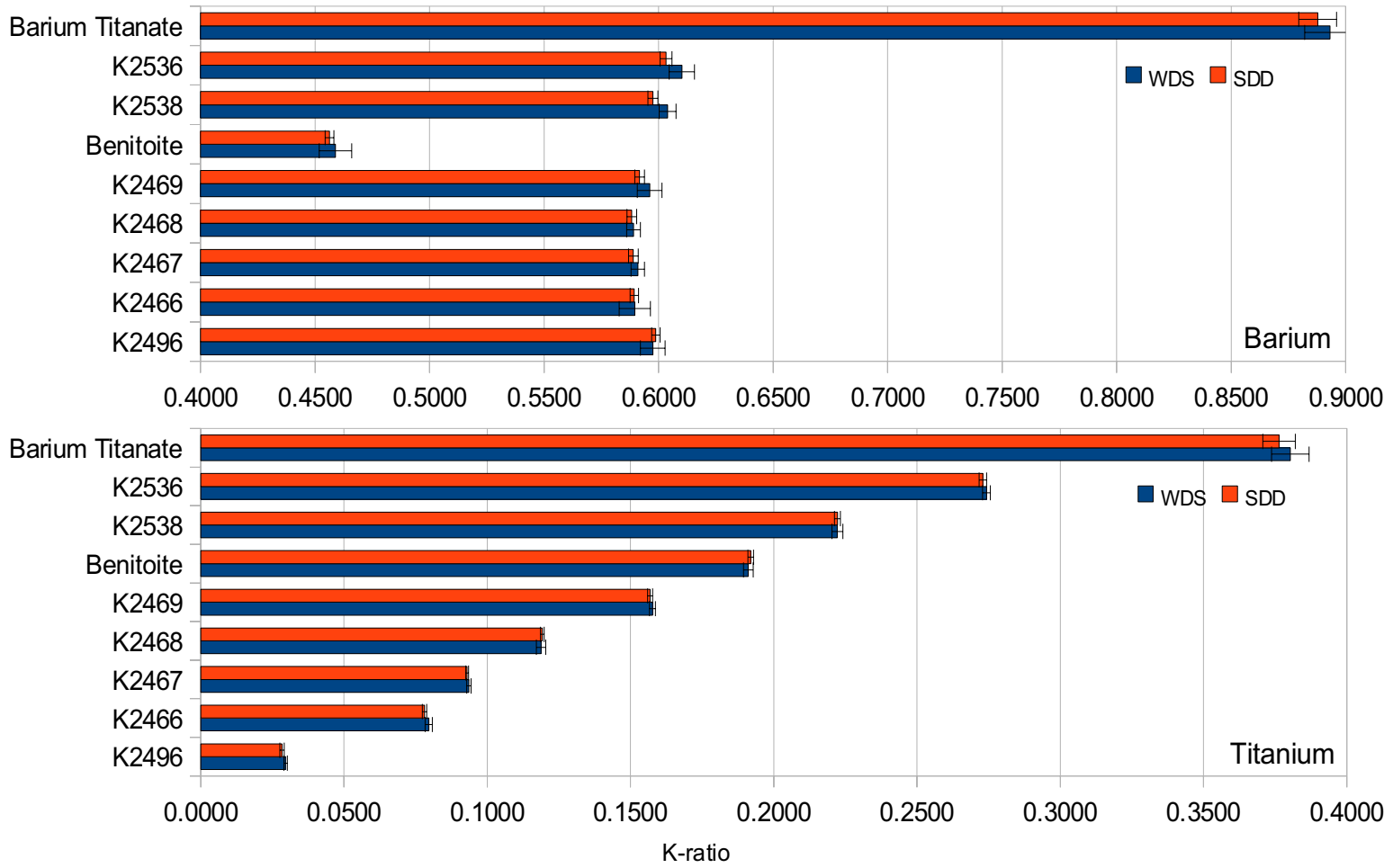

FIG. 1: K-ratios measured by wavelength and silicon drift spectrometers on a series of glasses and minerals containing Ba and Ti. 